\title{
DETERMINAÇÃO SIMULTÂNEA DE OLANZAPINA E CLORIDRATO DE FLUOXETINA EM FORMULAÇÕES FARMACÊUTICAS POR ESPECTROFOTOMETRIA DERIVATIVA
}

\author{
Jacira Izidório de Moura e Graziella Ciaramella Moita* \\ Departamento de Química, Centro de Ciências da Natureza, Universidade Federal do Piauí, 64049-550 Teresina - PI, Brasil
}

Recebido em 13/7/11; aceito em 18/9/11; publicado na web em 6/12/11

\begin{abstract}
SIMULTANEOUS DETERMINATION OF OLANZAPINE AND FLUOXETINE HYDROCHLORIDE IN PHARMACEUTICAL FORMULATIONS BY DERIVATIVE SPECTROPHOTOMETRY. Simple, sensitive and accurate spectrophotometric derivative methods were developed for the simultaneous determination of olanzapine and fluoxetine hydrochloride in pharmaceutical formulations by derivative spectrophotometry. On all orders of derivative studied, the linear response range was 10 to $60 \mathrm{mg} \mathrm{L}^{-1}$, with limit of quantitation (LoQ) ranging from 0.73 to $1.49 \mathrm{mg} \mathrm{L}^{-1}$ for fluoxetine hydrochloride and from 0.18 to $0.96 \mathrm{mg} \mathrm{L}^{-1}$ for olanzapine. The best orders for derivative analyses showed recoveries ranging from 99 to $103 \%$ and from 98 to $100 \%$, and inter-day accuracy $\leq$ $2.1 \%$ and $\leq 2.8 \%$, for fluoxetine hydrochloride and olanzapine, respectively.
\end{abstract}

Keywords: fluoxetine hydrochloride; olanzapine; simultaneous determination.

\section{INTRODUÇÃO}

A depressão é uma doença que atinge mais de 400 milhões de pessoas e, segundo estatísticas, quase $20 \%$ da população mundial passará pelo menos por um episódio de depressão ao longo da vida e, desses casos, quase metade terá repetidas crises e precisará de tratamento contínuo, o que leva a Organização Mundial de Saúde (OMS) a presumir que em 20 anos ela passará a ser a segunda doença mais dispendiosa e fatal. ${ }^{1-3}$

O cloridrato de fluoxetina (cloridrato de N-metil- $\gamma$-[4(trifluorometil)fenoxi]-benzonopropamina), é um inibidor seletivo de recaptação da serotonina, mundialmente utilizado no tratamento de depressão, ${ }^{1}$ síndromes de compulsão e do pânico e bulimia nervosa, ${ }^{4}$ por ser mais aceitável em termos de tolerância e toxicidade. ${ }^{1}$

Os casos que envolvem depressão associada a transtorno bipolar são ainda mais graves, pois o paciente passa por estados de extrema euforia, manias e profunda depressão. Para o tratamento desses casos a Eli Lilly and Company lançou o comprimido Symbyax ${ }^{\circledR}$, primeiro e único aprovado com sucesso pela FDA (Food and Drug Administration) em dezembro de 2003, que consiste na combinação do cloridrato de fluoxetina com o antipsicótico atípico olanzapina, ${ }^{5}$ 2-metil-4-(4-metil-1-piperazinil)-10H-tieno(2,3-b)(1,5) benzodiazepina, pertencente à classe das tioenobenzodiazepinas. ${ }^{6} \mathrm{~A}$ olanzapina é utilizada no tratamento da esquizofrenia e outras síndromes relacionadas, como transtorno bipolar do tipo I, sendo mais eficaz que outros psicotrópicos por levar a uma melhor resposta sem os efeitos colaterais apresentados por outros princípios ativos. ${ }^{6-8}$

O cloridrato de fluoxetina e olanzapina juntos atuam de forma sinérgica, pois aumentam os níveis dos três principais neurotransmissores (serotonina, dopamina e noradrenalina) que, quando em níveis baixos, podem provocar depressão. ${ }^{8}$ Assim, a atuação das duas drogas torna-se mais eficaz quando comparada com qualquer dos componentes isolados.

A literatura científica apresenta vários métodos para quantificação de olanzapina em formulações farmacêuticas, dentre eles, métodos utilizando técnicas espectrofotométricas no $\mathrm{UV}^{6}$ e no visível, ${ }^{9}$ cromatográfico, ${ }^{10}$ injeção em fluxo ${ }^{11}$ e métodos cinéticos. ${ }^{12}$ Para análise do cloridrato de fluoxetina há vários trabalhos que apresentam métodos

*e-mail: graziella@ufpi.edu.br usando espectrofotometria nas regiões do $U^{1}{ }^{1}$ e visível com formação de complexos, ${ }^{13,14}$ técnicas eletroquímicas, ${ }^{15}$ turbidimétricas ${ }^{16}$ e cromatografia líquida de alta eficiência (CLAE) para sua determinação em medicamentos, sendo esta última a técnica oficial apresentada nas Farmacopeias Americana e Brasileira. ${ }^{1}$

Para análise dos fármacos em formulações farmacêuticas contendo a combinação olanzapina e cloridrato de fluoxetina a técnica empregada é CLAE, ${ }^{4,17,18}$ que apresenta alguns inconvenientes como o uso de equipamentos caros e procedimentos complexos, o que torna o método pouco acessível e, em alguns casos, inviáveis.

O estudo da espectrofotometria derivativa, uma técnica simples para determinação simultânea de misturas binárias e ternárias, vem sendo foco de interesse nesses últimos anos, pois é útil em análises de misturas de componentes com espectros sobrepostos, possibilitando a quantificação de um ou mais analitos, sem prévia separação ou purificação dos mesmos. ${ }^{19,20}$ Os métodos utilizando esta técnica tornam-se, então, interessantes para farmácias de manipulação que desejam métodos que sejam rápidos, simples e de baixo custo, para quantificação de misturas binárias, que apresentam bandas de absorção sobrepostas no UV-visível como, por exemplo, o cloridrato de fluoxetina e olanzapina. Embora a associação destes fármacos não seja ainda comercializada no Brasil, as farmácias de manipulação podem produzir essa combinação com o diferencial de manipular fármacos com dosagens que atendam a necessidade individualizada de cada paciente. Neste sentido, o objetivo deste trabalho foi desenvolver um método utilizando a espectrofotometria derivativa para determinação simultânea da mistura olanzapina e cloridrato de fluoxetina em formulações farmacêuticas.

\section{PARTE EXPERIMENTAL}

\section{Materiais e reagentes}

Foram utilizadas amostras comerciais dos comprimidos de olanzapina $10 \mathrm{mg}$ (Zyprexa $\left.{ }^{\circledR}\right)$, cápsulas de cloridrato de fluoxetina $20 \mathrm{mg}$ (Fluxene ${ }^{\circledR}$ ) e os padrões de referência de olanzapina $(100,7 \%$, Gamma $^{\circledR}$ ), lote: 050201 e cloridrato de fluoxetina (100,1\%, Pharma nostra $\left.{ }^{\circledR}\right)$, lote: $10010342 \mathrm{~d}$.

Os reagentes utilizados foram todos de grau analítico: etanol (Nulear, Synth, Vetec), utilizado como solvente, ácido clorídrico e hidróxido de sódio (Vetec), usados para ajuste de $\mathrm{pH}$. 
Para o estudo de seletividade fez-se uso de excipientes aplicados rotineiramente em farmácias de manipulação, bem como nos dois medicamentos estudados: dióxido de silício coloidal 200 (Henrifar$\mathrm{ma}^{\circledR}$ ), croscarmelose sódica (Mingai ${ }^{\circledR}$ ), celulose microcristalina 102 $\left(\right.$ Pharma $\left.^{\circledR}\right)$, estearato de magnésio $\left(\right.$ Deg $\left.^{\circledR}\right)$, lactose malha $200\left(\right.$ Deg $\left.^{\circledR}\right)$ e talco $\left(\right.$ Pharma $\left.^{\circledR}\right)$.

\section{Equipamentos e parâmetros}

Utilizou-se um espectrofotômetro de duplo feixe Hitachi, U-3000, com os seguintes parâmetros de instrumento: intervalo de varredura 190 a $400 \mathrm{~nm}$, velocidade de varredura $300 \mathrm{~nm} \mathrm{~min}$, com leitura realizada a cada $0,5 \mathrm{~nm}$, abertura de fenda de $2,0 \mathrm{~nm}$ e lâmpada de deutério. Foram utilizadas cubetas de quartzo de $1 \mathrm{~cm}$ de caminho ótico e apenas solvente como branco. Para as medidas de massas fez-se uso de uma balança analítica Denver Instrument (APX 200) e usou-se o pHmetro Quimis (Q400AS) para verificação do pH. As suspensões das amostras foram centrifugadas em centrífuga Quimis (Q-222 TM).

\section{Amostras}

Foram utilizados comprimidos comerciais de olanzapina $10 \mathrm{mg}$ (Zyprexa $^{\circledR}$ ) e cápsulas de cloridrato de fluoxetina $20 \mathrm{mg}$ (Fluxene ${ }^{\circledR}$ ) para o preparo da amostra do fármaco associado. As fórmulas farmacêuticas foram pesadas, trituradas e homogeneizadas, a fim de se obter uma mistura com proporção $10 / 20 \mathrm{mg}$ de olanzapina e cloridrato de fluoxetina, respectivamente. Para o preparo de outra amostra do fármaco associado manipularam-se cápsulas contendo os dois princípios ativos com proporção 5/20 mg de olanzapina e cloridrato de fluoxetina, respectivamente. As duas amostras foram utilizadas nos ensaios de exatidão e nos ensaios de precisão apenas as amostras manipuladas foram utilizadas.

\section{Método}

O desenvolvimento do método analítico teve como base o guia da ANVISA com resolução RE nº 899 de 29 de maio de 2003, que determina que as figuras de mérito seletividade, linearidade, precisão, exatidão, limites de detecção e de quantificação e robustez devem ser analisadas para validação de métodos espectrofotométricos. ${ }^{21}$

\section{Seletividade}

Para avaliar se os excipientes interferem na determinação das substâncias em estudo, realizou-se a varredura espectral da suspensão contendo os principais excipientes contidos em ambas as drogas. As quantidades de excipientes foram calculadas com base em um comprimido contendo $5 \mathrm{mg}$ de olanzapina e $20 \mathrm{mg}$ de cloridrato de fluoxetina, resultando nas seguintes massas: $115,75 \mathrm{mg}$ de celulose microcristalina 102; 3,75 mg de croscarmelose sódica; $2,5 \mathrm{mg}$ de dióxido de silício coloidal; 2,5 mg de estearato de magnésio; 156,0 mg de lactose anidra e $5 \mathrm{mg}$ de talco. A suspensão do placebo foi preparada com concentrações de excipientes proporcionais às soluções de olanzapina a $5 \mathrm{mg} \mathrm{L}^{-1}$ e cloridrato de fluoxetina a $20 \mathrm{mg} \mathrm{L}^{-1}$. O espectro da suspensão foi derivado e comparado aos dos fármacos derivados na mesma ordem e variação de comprimento de onda $(\Delta \lambda)$.

\section{Linearidade}

A partir de soluções estoque de olanzapina e cloridrato de fluoxetina a $100 \mathrm{mg} \mathrm{L}^{-1}$ prepararam-se soluções, de cada fármaco, com concentrações entre 10 e $80 \mathrm{mg} \mathrm{L}^{-1}$ para a substância em análise e $10 \mathrm{mg} \mathrm{L}^{-1}$ fixa para o outro fármaco. Fez-se a varredura espectral das soluções e, em seguida, derivaram-se os espectros na $1^{\mathrm{a}}, 2^{\mathrm{a}}, 3^{\mathrm{a}} \mathrm{e}$ $4^{\mathrm{a}}$ ordem, com $\Delta \lambda$ variando de $1 \mathrm{a} 10 \mathrm{~nm}$, utilizando-se o programa computacional UV00 do espectrofotômetro. Construíram-se curvas de calibração para os dois fármacos plotando-se os valores dos sinais analíticos obtidos pelo método zero crossing ou ratio spectra em função da concentração. A partir dos resultados foi possível escolher a melhor ordem de derivada e $\Delta \lambda$ para as análises.

\section{Limites de detecção e quantificação}

Para avaliar os limites de detecção (LD) e quantificação (LQ) foram obtidos 21 espectros do branco e derivados na mesma ordem e $\Delta \lambda$ que os fármacos analisados. Em seguida, utilizou-se as expressões $\mathrm{LD}=3,3 \mathrm{~s} / \mathrm{b}$ e LQ $=10 \mathrm{~s} / \mathrm{b}$, em que $s$ é a estimativa do desvio padrão do sinal do branco e $b$ é o coeficiente angular da curva de calibração. ${ }^{22}$

\section{Precisão}

A precisão dos métodos foi estudada em termos de repetitividade e precisão intermediária. ${ }^{23}$ Para ambas formas de cálculo de precisão, foram preparadas nove soluções da amostra com concentrações de 50 e $200 \mathrm{mg} \mathrm{L}^{-1}$ de olanzapina e cloridrato de fluoxetina, respectivamente. As soluções foram centrifugadas por um período de 15 min a 3600 rpm. ${ }^{6}$ Em seguida, foram diluídas a fim de se obter, em triplica, as concentrações de 3 e $12 \mathrm{mg} \mathrm{L}^{-1} ; 8$ e $32 \mathrm{mg} \mathrm{L}^{-1} ; 12$ e 48 $\mathrm{mg} \mathrm{L}^{-1}$ para olanzapina e cloridrato de fluoxetina, respectivamente. Os resultados foram expressos em termos de desvio padrão relativo (DPR). Avaliou-se a concordância entre os resultados através do teste $F$ e teste $t$ pareado $(\mathrm{p}=0,05)$.

\section{Exatidão}

A exatidão foi determinada por intermédio do método de adição e recuperação de quantidades conhecidas de padrões de cloridrato de fluoxetina e olanzapina, correspondentes a 50, 100 e 150\% do valor de cada fármaco na amostra. ${ }^{23}$ Após adição de solução padrão à amostra, centrifugaram-se as suspensões obtidas por um período de 15 min a 3600 rpm. ${ }^{6}$ Em seguida, retiraram-se alíquotas do sobrenadante para o preparo das soluções a serem analisadas. As análises foram realizadas em triplicata e a exatidão foi expressa em porcentagem de recuperação.

\section{Efeito da marca do solvente}

Realizou-se teste de adição e recuperação de padrão para ambos os fármacos, com adição de $100 \%$ do valor correspondente a cada fármaco na amostra e utilizando-se etanol de três marcas diferentes (Synth, Nuclear e Vetec). As análises foram feitas em triplicata e expressas em termo de porcentagem recuperada. ${ }^{1}$

\section{Efeito da variação pH sobre o espectro de absorção}

Foram preparadas soluções de ácido clorídrico e hidróxido de sódio a $0,1 \mathrm{~mol} \mathrm{~L}^{-1}$ para avaliar o efeito do $\mathrm{pH}$ sobre os espectros de absorção de cloridrato de fluoxetina a $40 \mathrm{mg} \mathrm{L}^{-1}$ e olanzapina a $10 \mathrm{mg} \mathrm{L}^{-1}$. Adicionaram-se a 6 balões de $25 \mathrm{~mL}$, com micropipetas, os volumes de ácido ou base desejados e, depois, adicionou-se água suficiente de forma que o volume total de água na solução se mantivesse igual a $5 \mathrm{~mL}$ em todos os balões, e acertou-se o menisco à marca, usando etanol como solvente.

\section{Estudo da estabilidade}

Uma solução de cloridrato de fluoxetina a $40 \mathrm{mg} \mathrm{L}^{-1} \mathrm{em}$ etanol foi estocada em três frascos de mesmo volume, sendo um frasco transparente e os outros âmbares. $\mathrm{O}$ frasco transparente ficou exposto à luz direta, e um dos âmbares foi armazenado à temperatura ambiente, 
cerca de $25,0 \pm 4,0^{\circ} \mathrm{C}$, e o outro em geladeira a uma temperatura média de $3,9 \pm 0,2{ }^{\circ} \mathrm{C}$. Foi feita a varredura espectral das soluções a cada $30 \mathrm{~min}$, por um período de $3 \mathrm{~h}$. Cada solução também foi analisada após 8, 24 e $36 \mathrm{~h}$. O mesmo procedimento foi seguido para olanzapina a $10 \mathrm{mg} \mathrm{L}^{-1}$. Os espectros de ordem zero foram avaliados pelo teste ANOVA $(\mathrm{p}=0,05)$ em cada $\lambda_{\max }$ e em mais 20 pontos experimentais ao redor do $\lambda_{\max }$.

\section{RESULTADOS E DISCUSSÃO}

O comportamento espectral do cloridrato de fluoxetina e da olanzapina na faixa de concentração $10-60 \mathrm{mg} \mathrm{L}^{-1}$ foi estudado em solvente etanol (Figura 1) e na mistura etanol e água (1:1) (Figura 2), uma vez que ambas as drogas são pouco solúveis em água, porém facilmente solúveis em etanol. ${ }^{1,6}$

Utilizando-se apenas etanol como solvente, observam-se bandas mais bem definidas, permitindo a seleção de $\lambda_{\text {máx }}$ mais confiáveis para análise. Neste solvente cada fármaco apresenta três bandas de absorção máxima, sendo que para o cloridrato de fluoxetina há duas bandas

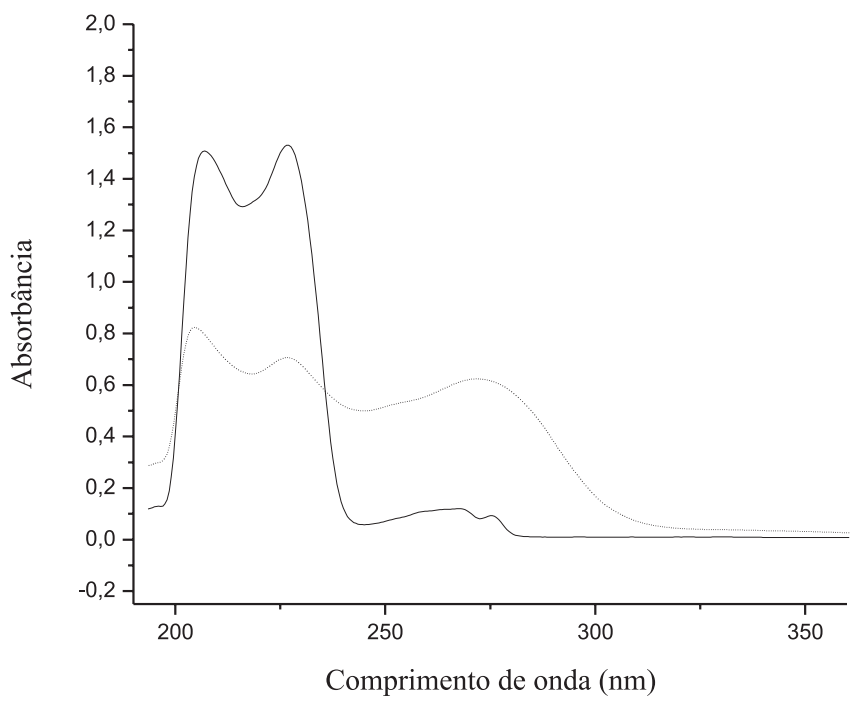

Figura 1. Espectros de absorção de ordem zero da olanzapina (----) a $10 \mathrm{mg}$ $L^{-1}$ e cloridrato de fluoxetina (-) a $40 \mathrm{mg} \mathrm{L}^{-1}$ em etanol

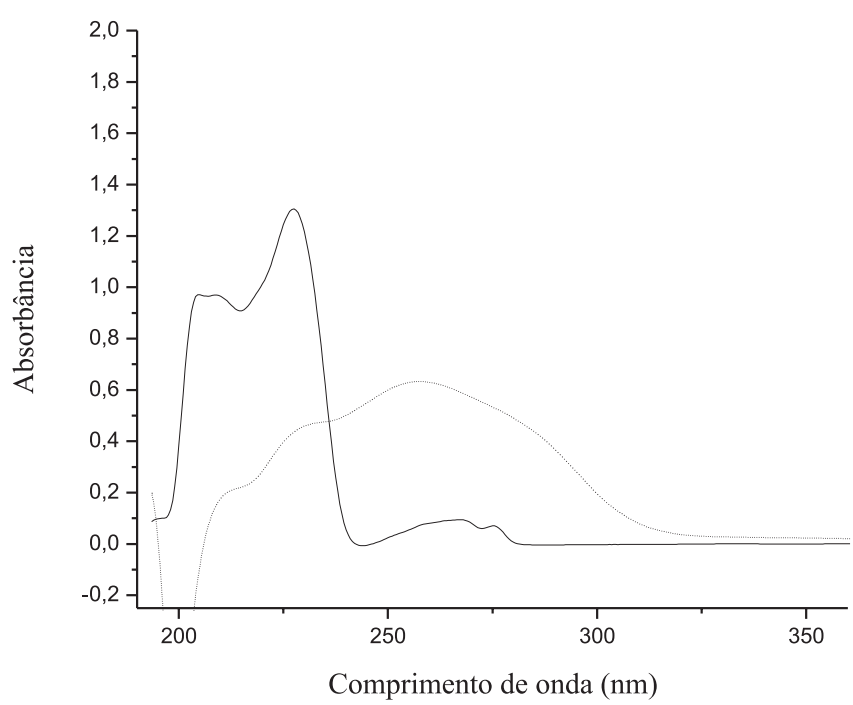

Figura 2. Espectros de absorção de ordem zero da olanzapina (----) a $10 \mathrm{mg}$ $L^{-1}$ e cloridrato de fluoxetina (-) a $40 \mathrm{mg} \mathrm{L}^{-1}$ na mistura etanol e água (1:1) de alta intensidade e bem definidas, em 207,0 e 227,0 nm, e uma banda de baixa intensidade a $276,0 \mathrm{~nm},{ }^{1}$ sendo que para a olanzapina existem três máximos bem definidos em 208,5; 226,5 e 272,5 nm. ${ }^{6}$ As bandas próximas ao ultravioleta de vácuo foram desconsideradas para análise, pois além de estarem totalmente sobrepostas, nesta região o etanol já começa a absorver.

Apesar da mistura etanol e água (1:1) utilizada como solvente solubilizar bem os dois fármacos, ela provoca alterações no espectro da olanzapina diminuindo o detalhamento espectral, não permitindo, assim, a visualização de uma banda bem definida, enquanto que para o cloridrato de fluoxetina ocorre apenas uma diminuição na intensidade da banda próxima ao ultravioleta de vácuo. Assim, apenas o etanol foi utilizado como solvente.

Tanto em etanol quanto na mistura etanol e água, os espectros estão totalmente sobrepostos, inviabilizando uma análise quantitativa por espectrofotometria de ordem zero, uma vez que o espectro da mistura terá máximos de absorção com intensidade resultante da soma da absorção das duas substâncias. ${ }^{24}$ Logo, foi utilizada a espectrofotometria derivativa como uma alternativa para determinação simultânea dos fármacos.

\section{Seleção dos comprimentos de onda para análise}

Os espectros de ordem zero dos fármacos que foram derivados da primeira a quarta derivadas, variando $\Delta \lambda$ de 1 a $10 \mathrm{~nm}$, revelaram que é possível a análise de cloridrato de fluoxetina na quarta derivada e $\Delta \lambda=4 \mathrm{~nm}$ (Figura 3) e de olanzapina na terceira e quarta derivadas para $\Delta \lambda=10 \mathrm{~nm}$ (Figuras 4 e 5) utilizando-se o método zero crossing, em que a análise de um dos fármacos na mistura é realizada através da medida da amplitude do espectro derivado da mistura a vários comprimentos de onda, e obtenção dos valores das amplitudes obtidas apenas nos comprimentos de onda em que o espectro derivado do outro fármaco cruza o zero. Assim, a medida da amplitude é proporcional somente à concentração do composto analisado e a curva de calibração obtida é dada pela relação entre intensidade da amplitude versus concentração do fármaco. ${ }^{19,25}$

O cloridrato de fluoxetina também pode ser analisado na segunda derivada $\Delta \lambda=2 \mathrm{~nm}$ (Figura 6) pelo método ratio spectra, onde se mede a amplitude máxima ou mínima de um espectro derivado, obtido pela divisão do espectro da mistura dos fármacos por um espectro de concentração fixa da olanzapina. As misturas possuem

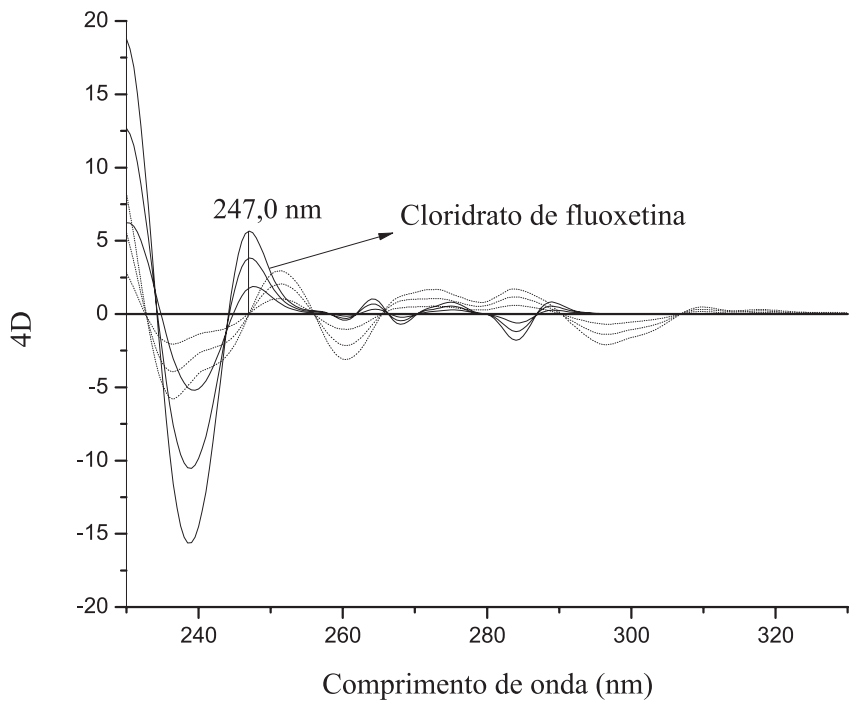

Figura 3. Espectros da quarta derivada $(\Delta \lambda=4)$ pelo método zero crossing de soluções de cloridrato de fluoxetina (-) e olanzapina (----) a 10, 20 e $30 \mathrm{mg} \mathrm{L}^{-1}$ 


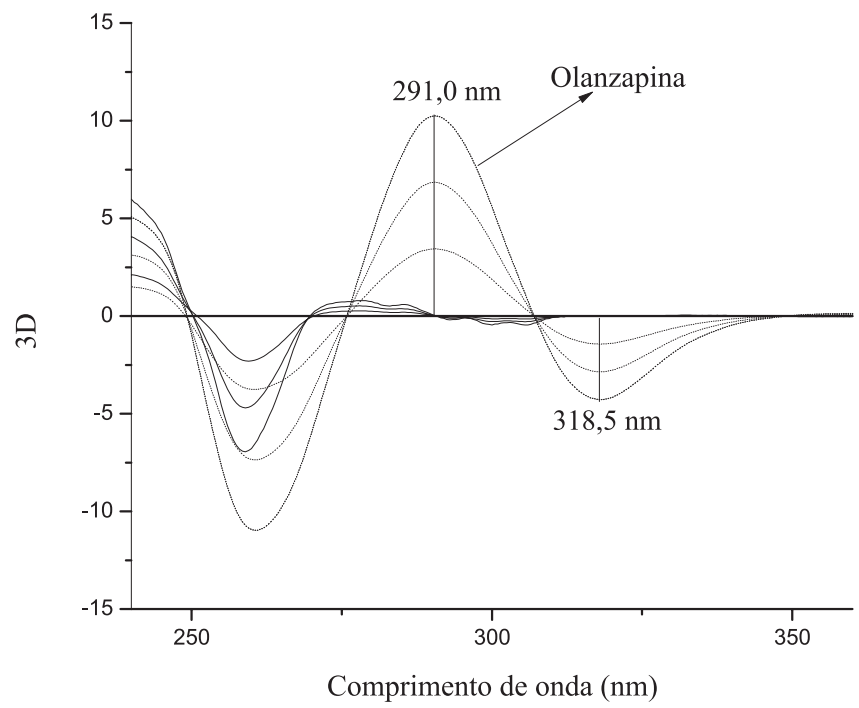

Figura 4. Espectros da terceira derivada $(\Delta \lambda=10)$ pelo método zero crossing de soluções de cloridrato de fluoxetina (-) e olanzapina (----) a 10, 20 e $30 \mathrm{mg} \mathrm{L}^{-1}$

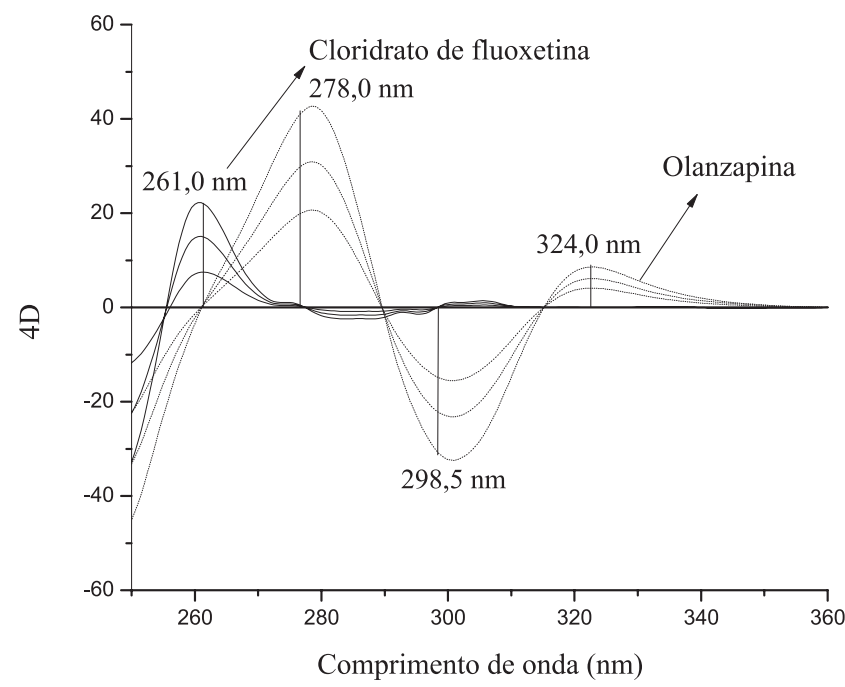

Figura 5. Espectros da quarta derivada $(\Delta \lambda=10)$ pelo método zero crossing de soluções de cloridrato de fluoxetina (-) e olanzapina (----) a 10, 20 e $30 \mathrm{mg} \mathrm{L}^{-1}$

concentrações variáveis de cloridrato de fluoxetina e concentração fixa de olanzapina, assim, as curvas de calibração são obtidas pela relação entre amplitudes obtidas, correspondentes apenas ao cloridrato de fluoxetina, por suas respectivas concentrações. ${ }^{19} \mathrm{~A}$ olanzapina não pode ser analisada por este método, na faixa de concentração estudada.

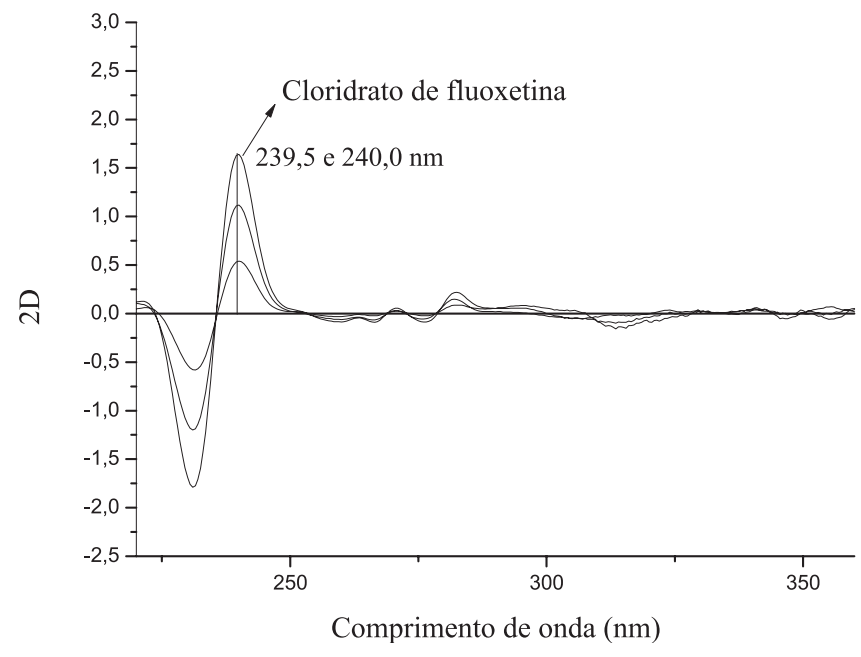

Figura 6. Espectros da segunda derivada $(\Delta \lambda=2)$ pelo método ratio spectra de soluções de cloridrato de fluoxetina a 10,20 e $30 \mathrm{mg} \mathrm{L}^{-1}$

Apesar do cloridrato de fluoxetina e olanzapina apresentarem comprimentos de onda com amplitudes máximas em 261,0 nm (Figura 5) e 291,0 nm (Figura 4), respectivamente, pelo método zero crossing, onde o outro fármaco cruza o zero em um ponto isosbéstico, as curvas de calibração obtidas não são adequadas para análise por apresentarem valores altos para o intercepto da curva e coeficiente de correlação linear inadequado $(<0,99)$. A olanzapina apresenta três comprimentos de onda na quarta derivada $\Delta \lambda=10$ (Figura 5) em que o cloridrato de fluoxetina cruza o zero em um ponto isosbéstico, no entanto, em 278,0 e 298,5 nm os interceptos das curvas de calibração obtidas também apresentaram valores altos.

A análise do cloridrato de fluoxetina foi realizada nos comprimentos de onda em 239,5 e 240,0 nm da segunda derivada (2D) (Figura 6) pelo método ratio spectra e $247,0 \mathrm{~nm}$ da quarta derivada (4D) (Figura 3) pelo método zero crossing, e da olanzapina nos comprimentos de onda em 318,5 nm da terceira derivada (3D) (Figura 4) e 324, $0 \mathrm{~nm}$ (Figura 5) da quarta derivada (4D) pelo método zero crossing.

Os parâmetros analíticos para análise simultânea dos dois fármacos estão expressos na Tabela 1. A curva de calibração (unidade derivativa versus concentração) pelo método ratio spectra foi obtida usando concentração do divisor de $10 \mathrm{mg} \mathrm{L}^{-1}$ de olanzapina. Para o método zero crossing, a concentração fixa do interferente foi de $10 \mathrm{mg} \mathrm{L}^{-1}$. Para os dois métodos a concentração do analito com resposta linear variou de 10 a $60 \mathrm{mg} \mathrm{L}^{-1}$.

Todas as curvas de calibração apresentaram boa linearidade dentro da faixa de concentração estudada, com coeficientes de correlação maiores que 0,999 , melhores que os obtidos em trabalhos prévios ${ }^{4}$ para olanzapina $(r=0,9992)$ e maiores ou iguais para cloridrato de fluoxetina $(0,9996)$, estando dentro da faixa estabelecida pela ANVISA $(r>0,99)$. Pela Tabela 1 pode-se perceber que o método zero

Tabela 1. Parâmetros analíticos para os métodos ratio spectra (2D) com divisor a $10 \mathrm{mg} \mathrm{L}^{-1}$ e zero crossing (3D; $\left.4 \mathrm{D}\right)$ com concentração fixa a 10 mg $\mathrm{L}^{-1}$

\begin{tabular}{|c|c|c|c|c|c|c|}
\hline Fármacos & Método $(\lambda, \mathrm{nm})$ & Equação & $\mathrm{r}$ & $\mathrm{LD}\left(\mathrm{mg} \mathrm{L}^{-1}\right)$ & $\mathrm{LQ}\left(\mathrm{mg} \mathrm{L}^{-1}\right)$ & $\mathrm{LL}\left(\mathrm{mg} \mathrm{L}^{-1}\right)$ \\
\hline FLU & $2 \mathrm{D}(239,5)$ & $-0,004+0,055 \mathrm{C}$ & 0,9999 & 0,46 & 1,27 & 60,0 \\
\hline FLU & $2 \mathrm{D}(240,0)$ & $0,022+0,054 \mathrm{C}$ & 0,9999 & 0,49 & 1,49 & 60,0 \\
\hline FLU & 4D $(247,0)$ & $0,187+0,184 C$ & 0,9996 & 0,24 & 0,73 & 60,0 \\
\hline OLZ & $3 \mathrm{D}(318,5)$ & $0,034-0,129 \mathrm{C}$ & $-0,9999$ & 0,06 & 0,18 & 60,0 \\
\hline OLZ & 4D $(324,0)$ & $-0,126+0,178 \mathrm{C}$ & 0,9998 & 0,32 & 0,96 & 60,0 \\
\hline
\end{tabular}

FLU - cloridrato de fluoxetina, OLZ - olanzapina, 2D, 3D e 4D - segunda, terceira e quarta ordem. $y=a+b x$ onde: $a$ - intercepto de $x$ com o eixo y e b - inclinação da reta. $r$ = coeficiente de correlação linear. LD - Limite de detecção, LQ - Limite de quantificação e LL - limite de linearidade 
crossing na 3D (318,5 nm) e 4D (247,0 $\mathrm{nm})$ apresentou os menores LD e LQ para olanzapina e cloridrato de fluoxetina, respectivamente. Os limites de quantificação foram comparados com os obtidos na literatura, ${ }^{4}$ que apresentaram os valores de $5 \times 10^{-3} \mathrm{mg} \mathrm{L}^{-1} \mathrm{e} 10^{-3} \mathrm{mg} \mathrm{L}^{-1}$ para olanzapina e cloridrato de fluoxetina, respectivamente. Embora os valores obtidos sejam maiores, estão abaixo do valor da menor concentração estudada neste trabalho.

\section{Estudo da seletividade}

O estudo da seletividade foi feito com uma combinação de alguns excipientes contidos em cada fármaco, e as concentrações usadas foram semelhantes às usualmente utilizadas em compridos manipulados. Em geral, os excipientes não apresentaram boa solubilidade em etanol, precipitaram ou ficaram suspensos espalhando a radiação, o que pode ter contribuído para um sinal analítico de baixa intensidade na região em torno de $208 \mathrm{~nm}$. As bandas de cloridrato de fluoxetina e olanzapina nesta região não são adequadas para análise, assim, não há interferência do placebo nesta região.

A pequena interferência do placebo nos outros máximos de absorção dos fármacos foi suavizada pela separação dos excipientes por centrifugação e decantação, bem como pelo uso da espectrofotometria derivativa, que permite uma diminuição no sinal dos interferentes (Figura 1S, material suplementar). ${ }^{6,26}$

\section{Efeito da marca do solvente}

Os métodos 2D (239,5 nm) e 3D $(318,5 \mathrm{~nm})$ foram utilizados para o estudo do efeito da marca do solvente, fazendo-se o teste de adição e recuperação de padrão para uma concentração, em triplicata, utilizando-se solventes de três marcas diferentes. Os resultados obtidos estão expressos na Tabela 2.

Tabela 2. Resultados da avaliação da recuperação dos padrões de olanzapina e cloridrato de fluoxetina utilizando-se etanol de diferentes marcas (A, B e C) e os métodos zero crossing e ratio spectra

\begin{tabular}{ccccc}
\hline \multicolumn{2}{c}{ Fármacos } & \multicolumn{3}{c}{ \% Recuperação (DPR) } \\
métodos $(\lambda, \mathrm{nm})$ & A & B & C \\
\hline OLZ & 3D $(318,5)$ & $100(1,1)$ & $100(4,7)$ & $100(1,7)$ \\
FLU & 2D $(239,5)$ & $100(2,2)$ & $99(0,5)$ & $99(1,7)$ \\
\hline
\end{tabular}

FLU - cloridrato de fluoxetina, OLZ - olanzapina, 2D e 3D - segunda e terceira ordem. DPR - desvio padrão relativo

A avaliação da robustez utilizando-se etanol de três marcas diferentes para os métodos em estudo revelou pelo teste ANOVA, a 95\% de confiança, que não há diferença significativa entre as recuperações obtidas com o uso das três marcas, nos dois métodos.

\section{Estudo da estabilidade}

O teste de estabilidade revelou que as soluções etanólicas de cloridrato de fluoxetina e olanzapina que foram estocadas sob refrigeração e em temperatura ambiente, ambas protegidas da incidência de luz, não apresentaram degradação química. As soluções estocadas sob exposição direta da luz, não sofreram degradação fotoquímica, no período em estudo, uma vez que os sinais analíticos permaneceram constante em cada $\lambda_{\max }$ do espectro de ordem zero, como mostrado na Figura 7. O teste de variância (ANOVA, $p=0,05$ ) aplicado confirmou que todas as soluções estocadas apresentaram absorbâncias estatisticamente iguais, no período em estudo. Na literatura ${ }^{4}$ são encontrados estudos sobre a estabilidade de soluções padrão das duas drogas em temperatura ambiente e protegidas da luz por 3 dias, e também não se observou degradação significativa neste período. No entanto, outros estudos ${ }^{6}$ indicam que a solução de olanzapina só é estável até o terceiro dia, na presença da luz, mas para as outras condições a estabilidade permanece por um mês.

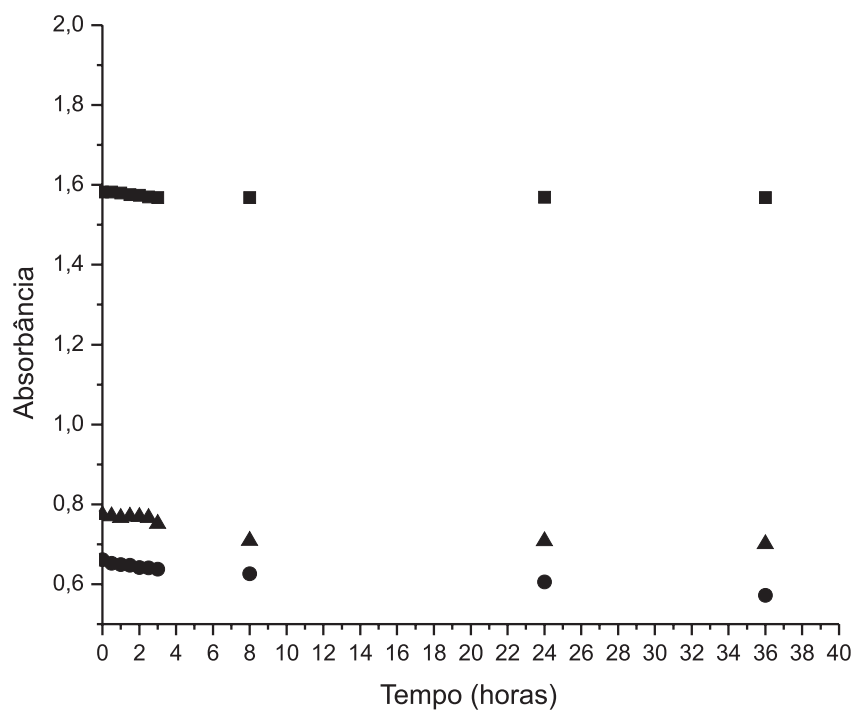

Figura 7. Efeito da luz sobre as soluções de cloridrato de fluoxetina $40 \mathrm{mg}$

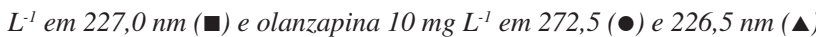

\section{Estudo do pH sobre o espectro de absorção}

O estudo do comportamento espectral de soluções de cloridrato de fluoxetina a $40 \mathrm{mg} \mathrm{L}^{-1} \mathrm{com} \mathrm{pH}$ variando entre 2,66 a 11,63, quando comparado ao de uma solução sem adição de tampão ( $\mathrm{pH}$ 8,72 ), revelou que em meio ácido ocorre uma acentuada diminuição na intensidade de absorção e uma mudança no perfil da banda em 201,0 nm, que fica mais aguda. Segundo Souter e Dinner, ${ }^{27}$ a ocorrência de uma degradação química significativa ocorre apenas em valores menores que $\mathrm{pH} 1$. Em meio básico o cloridrato de fluoxetina apresenta comportamento espectral similar ao de uma solução sem o tampão.

A solução etanólica de olanzapina apresenta pH 7,33 e o comportamento espectral de soluções a $10 \mathrm{mg} \mathrm{L}^{-1} \mathrm{em}$ meio ácido revela uma acentuada mudança no perfil quando comparado a uma solução sem adição de tampão, devido a uma possível reação do tipo ácido base. Em meio básico os espectros não apresentaram mudanças significativas quando comparados uma solução sem adição de tampão. ${ }^{6}$

\section{Precisão}

Os ensaios de precisão foram realizados apenas com as amostras manipuladas. Os resultados (Tabela 3) revelam que os métodos estudados apresentam boa repetitividade e precisão intermediária, com desvios padrão relativos variando entre 0,4 e 3,3\% para o cloridrato de fluoxetina e 0,3 e 2,8\% para olanzapina, valores inferiores ao máximo estabelecido pela ANVISA (5,0\%). ${ }^{21}$

As precisões intra e interdia foram comparadas através dos testes $F$ e $t$ pareado. Os resultados revelaram que para os três níveis de concentração estudados, os valores de $F$ encontrados $(1,47 ; 3,12$ e $4,58)$ são menores que o tabelado $(6,39)$, e que para o teste $t$ pareado $(\mathrm{p}=0,05)$ a comparação entre desvios para cada nível de concentração apresentou valores $(0,16 ; 1,76$ e 1,70$)$ menores que o tabelado $(2,77)$, assim, pelos dois testes não há diferença significativa entre os dois resultados. 
Tabela 3. Resultados do ensaio de precisão dos fármacos na mistura 4:1 em massa, utilizando os métodos ratio espectra e zero crossing e concentração fixa a $10 \mathrm{mg} \mathrm{L}^{-1}$

\begin{tabular}{|c|c|c|c|c|c|c|}
\hline \multirow{2}{*}{\multicolumn{2}{|c|}{ Concentração (mg L-1) }} & \multicolumn{5}{|c|}{$\operatorname{DPR}(\%)$} \\
\hline & & \multicolumn{5}{|c|}{ Precisão intradia (Analista 1) } \\
\hline OLZ & FLU & FLU $(239,5)$ & FLU $(240,0)$ & FLU $(247,0)$ & OLZ $(318,5)$ & OLZ $(324,0)$ \\
\hline 3 & 12 & 1,3 & 1,6 & 1,8 & 1,3 & 2,4 \\
\hline 8 & 32 & 0,8 & 0,5 & 0,4 & 0,4 & 0,3 \\
\hline \multirow[t]{2}{*}{12} & 48 & 2,1 & 3,3 & 2,1 & 2,1 & 2,4 \\
\hline & & \multicolumn{5}{|c|}{ Precisão interdia (Analista 2) } \\
\hline OLZ & FLU & FLU $(239,5)$ & FLU $(240,0)$ & FLU $(247,0)$ & $\operatorname{OLZ}(318,5)$ & OLZ $(324,0)$ \\
\hline 3 & 12 & 1,2 & 1,2 & 2,1 & 1,5 & 2,5 \\
\hline 8 & 32 & 0,7 & 0,8 & 0,4 & 0,8 & 1,1 \\
\hline 12 & 48 & 0,4 & 0,7 & 0,6 & 2,8 & 2,4 \\
\hline
\end{tabular}

FLU - cloridrato de fluoxetina, OLZ - olanzapina, DPR - desvio padrão relativo

\section{Exatidão}

Os resultados do ensaio de exatidão, realizado em três níveis de concentração e em triplicata, utilizando comprimidos manipulados (Tabela 4), revelaram que todos os métodos apresentam recuperações dentro da faixa estabelecida pela ANVISA $(80-120 \%),{ }^{21}$ no entanto, o melhor método para análise do cloridrato de fluoxetina é o 2D (239,5 $\mathrm{nm})$ pelo ratio spectra, onde as recuperações variaram entre 99 a $103 \%$, e para olanzapina é o método $3 \mathrm{D}(318,5 \mathrm{~nm})$ com recuperações na faixa entre 99 a $100 \%$, sendo comparáveis aos resultados obtidos por outros autores ${ }^{4,17,18}$ que utilizaram a técnica de cromatografia líquida de alta eficiência.

Tabela 4. Resultados do teste de adição e recuperação dos padrões cloridrato de fluoxetina e olanzapina em três níveis de concentração

\begin{tabular}{lccc}
\hline $\begin{array}{l}\text { Fármacos } \\
\text { métodos }(\lambda, \mathrm{nm})\end{array}$ & $\begin{array}{c}\text { Adicionada } \\
\left(\mathrm{mg} \mathrm{L}^{-1}\right)\end{array}$ & $\begin{array}{c}\text { Recuperada } \\
\left(\mathrm{mg} \mathrm{L}^{-1}\right)\end{array}$ & $\begin{array}{c}\% \text { Recuperada } \\
(\mathrm{DPR})\end{array}$ \\
\hline $\begin{array}{l}\text { Fluoxetina } \\
\text { ratio spectra, 2D }\end{array}$ & 10 & 10,3 & $103(1,2)$ \\
$(239,5)$ & 20 & 20,1 & $101(2,2)$ \\
\hline Fluoxetina & 30 & 29,8 & $99(2,4)$ \\
ratio spectra, 2D & 10 & 10,5 & $105(1,2)$ \\
$(240,0)$ & 20 & 20,3 & $101(1,8)$ \\
\hline Fluoxetina & 30 & 30,0 & $100(2,9)$ \\
zero crossing, 4D & 10 & 10,8 & $108(1,6)$ \\
$(247,0)$ & 20 & 20,4 & $102(1,5)$ \\
\hline Olanzapina & 30 & 28,7 & $96(4,1)$ \\
zero crossing, 3D & 10 & 9,9 & $99(2,4)$ \\
$(318,5)$ & 20 & 19,9 & $99(1,1)$ \\
& 30 & 29,5 & $98(1,5)$ \\
\hline Olanzapina & 10 & 9,4 & $94(2,9)$ \\
zero crossing, 4D & 20 & 19,3 & $96(1,2)$ \\
(324,0) & 30 & 28,5 & $95(1,7)$ \\
\hline
\end{tabular}

2D, 3D, 4D - Segunda, terceira e quarta ordem. DPR - desvio padrão relativo

A recuperação também foi aplicada para misturas homogêneas dos comprimidos de cada fármaco e variaram entre 96 a $105 \%$ para o cloridrato de fluoxetina e 96 a 104\% para olanzapina, ficando dentro da faixa estabelecida pela ANVISA. Os métodos zero crossing 4D $(247,0)$ e ratio spectra $2 \mathrm{D}(239,5)$ para análise da fluoxetina foram aplicados a uma amostra e comparados pelos testes $F$ e $t$ pareado. Pelo teste $F$ as medidas são precisas $\left(\mathrm{f}_{\text {cal }} 1,07<\mathrm{F}_{\text {tab }} 19,0\right)$ e de acordo com o teste $t$ pareado $(\mathrm{p}=0,05)$ os métodos são estatisticamente iguais $\left(\mathrm{t}_{\text {cal }} 0,748<\mathrm{t}_{\text {tab }} 4,303\right)$.

\section{CONCLUSÃO}

Os métodos desenvolvidos para determinação simultânea de cloridrato de fluoxetina e olanzapina são seletivos e robustos quanto aos fatores variação de temperatura, marca do solvente e pH básico, e lineares na faixa de concentração estudada. A exatidão dos métodos apresentou valores de recuperação semelhantes aos já obtidos por CLAE e a precisão, valores abaixo do máximo estabelecido pela ANVISA. Assim, os métodos podem ser rotineiramente usados na quantificação dos fármacos, uma vez que são simples, não requerem prévia separação dos fármacos e são economicamente mais viáveis que os métodos utilizando técnicas cromatográficas, já utilizadas na análise simultânea das drogas.

\section{MATERIAL SUPLEMENTAR}

No material suplementar, disponível em http://quimicanova.sbq. org.br, com acesso livre e em arquivo PDF, encontra-se a Figura 1S, apresentando os espectros derivados do cloridrato de fluoxetina, da olanzapina e do placebo, para comparação.

\section{AGRADECIMENTOS}

Ao CNPq, pela bolsa de mestrado concedida, à Farmácia de Manipulação Galeno por ceder a matéria-prima do cloridrato de fluoxetina e os excipientes, à farmacêutica A. K. M. F. Lustosa pela orientação quanto à manipulação das cápsulas, e aos alunos de iniciação I. J. G. da Silva e A. C. dos S. Costa pela colaboração nos ensaios de precisão.

\section{REFERÊNCIAS}

1. Fregonezi-Nery, M. M.; Baracat, M, M.; Casagrande, R.; Machado, T. M.; Miglioranza, B.; Gianotto, E. A. S.; Dalmas, J. C.; Quim. Nova 2008, 31, 1665 .

2. http://gballone.sites.uol.com.br/news16.htm, acessada em Setembro 2011.

3. Thase, M. E.; J. Clin. Psychiatry 1999, 60, 3.

4. Reddy, B. V.; Reddy, K. V. N.; Sreeramulu, J.; Kanumula, G. V.; Chromatographia 2007, 66, 111. 
5. http://www.lilly.com/pdf/symbyax_factsheet_revised062905.pdf, acessada em Setembro 2011.

6. Rêgo, J. F.; Moura, J. I.; Moita, G. C.; Quim. Nova 2010, 33, 471.

7. Lacerda, A. L. T.; Soares, J. C.; Tohen, M.; Rev. Bras. Psiquiatr. 2002, 24,34 .

8. http://www.theodora.com/drugs/symbyax_capsules_lilly.html, acessada em Setembro 2011.

9. Rajendraprasad, N.; Basavaiah, K.; J. Anal. Chem. 2010, 65, 482.

10. Patel, R, B.; Patel, M. R.; Bhatt, K. K.; Patel, B. G.; J. AOAC Int. 2010 93, 811.

11. Jasiñka, A.; Nalewajko, E.; Anal. Chim. Acta 2004, 508, 165.

12. Mohamed, A. A.; Monatsh. Chem. 2008, 139, 1005.

13. Prabhakar, A. H.; Patel, V. B.; Giridhar, R.; J. Pharm. Biomed. Anal. 1999, 20, 427.

14. Bebawy, L. I.; El-Kousy, N.; Suddik, J. K.; Shokry, M.; J. Pharm. Biomed. Anal. 1999, 21, 133.

15. Hussien, E. M.; Abdel-Gawad, F. M.; Issa, Y. M.; Biochem. Eng. J. 2011, 53,210 .

16. Suarez, W. T.; Sartori, E. R.; Batista, E. F.; Fatibello-Filho, O.; Quim. Nova 2009, 32, 2396.
17. Shah, C. R.; Shah, N. J.; Suhagia, B. N.; Patel, N. M.; J. AOAC Int. 2007, 90, 1573.

18. Patel, S.; Patel, N. J.; Indian J. Pharm. Sci. 2009, 71, 477.

19. El-Sayad, A. Y.; El-Salem, N. A.; Anal. Sci. 2005, 21, 595.

20. Paschoal, L. R.; Ferreira, W. A.; Prado, M. R. D.; Vilela, A. P. O.; Rev. Bras. Ciênc. Farm. 2003, 39, 105.

21. Agência Nacional de Vigilância Sanitária (ANVISA); Resolução RE $n^{o}$ 899, de 29/05/2003, publicada em 2/6/2003.

22. Leite, F.; Validação em Análise Química, 4a ed., Editora Átomo: Campinas, 2002.

23. Ribani, M.; Botolli, C. B. G.; Collins, C. H.; Jardim, I C. S. F.; Melo, L. F. C.; Quim. Nova 2004, 27, 771.

24. García, J. M.; Hernández, O.; Jiménez, A. I.; Jiménez, F.; Arias, J. J.; Anal. Chim. Acta 1995, 317, 83.

25. Karpinska, J.; Talanta 2004, 64, 801.

26. Mendham, J.; Denney, R. C.; Baernes, J. D.; Thomas, M. J. K.; Vogel: Análise Química Quantitativa, 6a ed., Editora LTC: Rio de Janeiro, 2002.

27. Souter, R. W.; Dinner, A.; J. Pharm Sci. 1976, 65, 457. 


\section{DETERMINAÇÃO SIMULTÂNEA DE OLANZAPINA E CLORIDRATO DE FLUOXETINA EM FORMULAÇÕES FARMACÊUTICAS POR ESPECTROFOTOMETRIA DERIVATIVA}

Jacira Izidório de Moura e Graziella Ciaramella Moita*

Departamento de Química, Centro de Ciências da Natureza, Universidade Federal do Piauí, 64049-550 Teresina - PI, Brasil
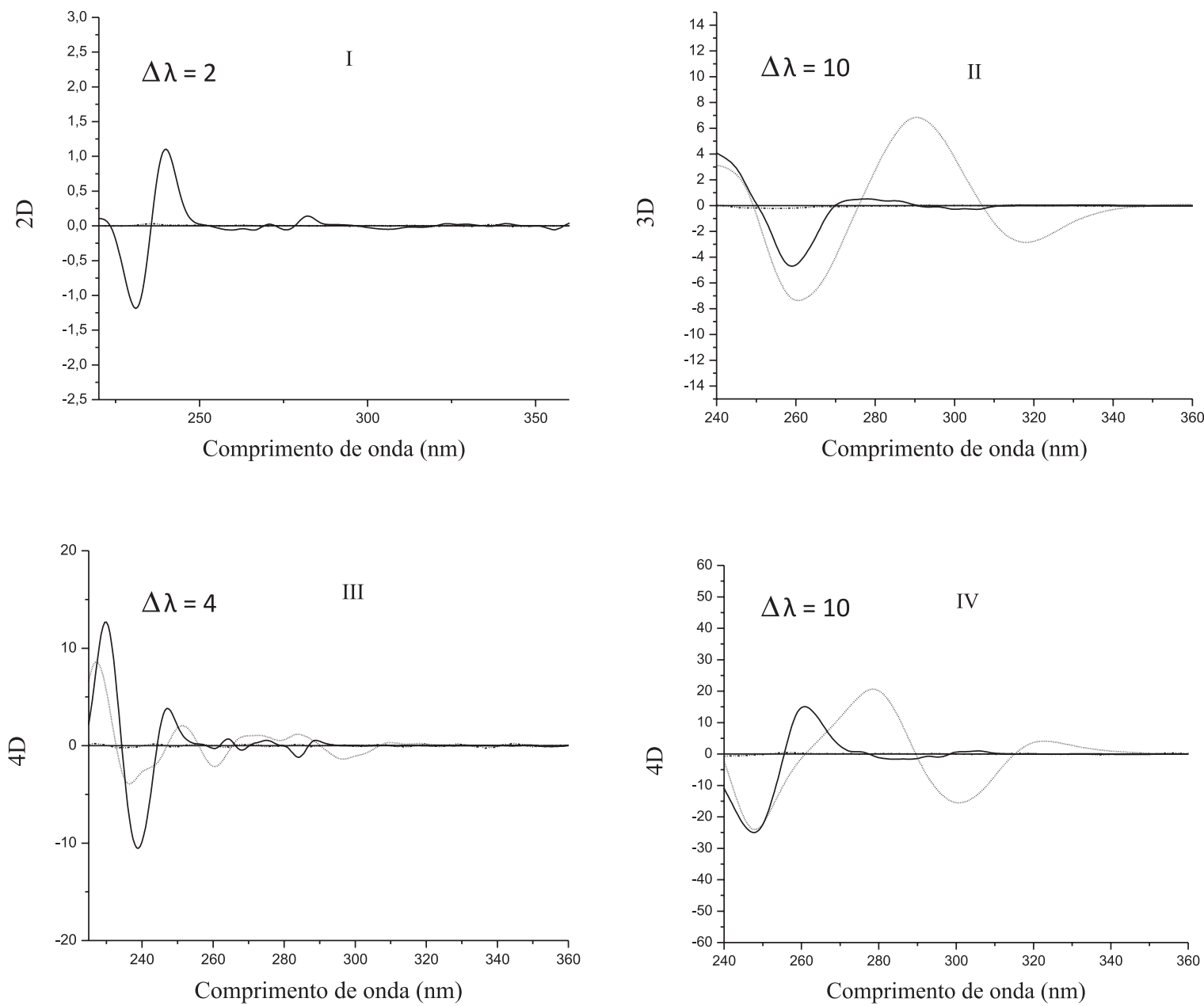

Figura 1S. Espectros da segunda derivada (I) pelo método ratio spectra e da terceira (II) e quarta derivada (III e IV) pelo método zero crossing de soluções de

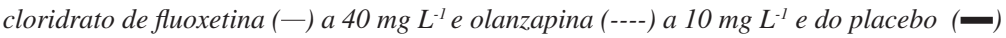

